\title{
Snorkel and chimney endografting for juxtarenal and thoracoabdominal aortic aneurysms is an acceptable, but aging technology
}

\author{
Zachary S. Pallister ${ }^{1}$, Ross Milner ${ }^{2}$, Jayer Chung ${ }^{1}$ \\ ${ }^{1}$ Division of Vascular Surgery and Endovascular Therapy, Michael E DeBakey Department of Surgery, Baylor College of Medicine, Houston, TX, \\ USA; ${ }^{2}$ Section of Vascular and Endovascular Surgery, Department of Surgery, Pritzker School of Medicine, University of Chicago, Chicago, IL, USA \\ Correspondence to: Jayer Chung, MD. Division of Vascular Surgery and Endovascular Therapy, Michael E DeBakey Department of Surgery, Baylor \\ College of Medicine, One Baylor Plaza, MS 390, Houston, TX 77030, USA. Email: Jayer.Chung@bcm.edu.
}

Submitted Apr 27, 2021. Accepted for publication Oct 05, 2021.

doi: $10.21037 /$ acs-2021-taes-14

View this article at: https://dx.doi.org/10.21037/acs-2021-taes-14

Due to the efficacy and safety of endovascular aortic repair (EVAR) in the infrarenal aorta, interventionalists have expanded the application of EVAR for juxtarenal, paravisceral and type IV thoracoabdominal aortic aneurysms (TAAA). One example uses snorkel/periscope/and/or chimney (ChEVAR) techniques for exclusion of TAAA aneurysms. These techniques were initially reported in 2003 (1) and were primarily used in juxtarenal aneurysms with inadequate infrarenal necks. 'Chimney' refers to visceral stents with inflow from the aorta proximal to the main aortic graft with antegrade perfusion to the target vessels. Chimneys are placed via an antegrade access from the arm. Alternatively, 'snorkels' or 'periscopes' refer to the use of stents that receive their inflow distal to the main aortic stent graft and perfuse the target vessel in a retrograde manner. The chimney/snorkels frequently are covered balloon expandable stents, though self-expanding covered stents are also utilized. ChEVAR contrasts with fenestrated/branched EVAR (FEVAR) which utilize customized fenestrations and/or branches within the body of the graft to extend the seal of the endograft whilst preserving branch vessel flow. Herein, we will briefly discuss the merits and pitfalls of ChEVAR for the management of juxtarenal and type IV TAAA.

\section{Advantages}

Off-the-shelf availability remains the primary advantage of ChEVAR. This obviates delays associated with manufacturing fenestrated endografts. This is most valuable for symptomatic, rapidly expanding or ruptured aneurysms. The device caliber necessary to treat a given pathology is frequently smaller with ChEVAR. This is important for patients with narrow, calcified and/or tortuous iliac access. ChEVAR requires experience and instruction, but does not necessitate the acquisition of entirely new skills of cannulating fenestrations/branches.

Medico-legal aspects of these repairs are also essential to consider. The Z-Fen device (Cook Medical, USA) is the only fenestrated device approved by the Food and Drug Administration (FDA). Other fenestrated/branched endografts, especially physician modified endografts (PMEG), are labeled by the FDA as 'significant-risk devices' that require significant regulatory oversight. Ideally, these fenestrated/branched endografts require investigational device exemptions (IDE) from the FDA. In contrast, an IDE is not required for ChEVAR procedures.

\section{Disadvantages}

Gaps frequently persist between the aortic stent graft and the branch vessel stents. These are loosely defined as 'gutter leaks'. These leaks are especially concerning because neither the endografts nor branch stent grafts have been specifically engineered for ChEVAR. Theoretically, the chimneys and snorkels are placed parallel to the long axis of the aortic neck, which is the direction stents will form an optimal wrap around the branch stent. In reality, however, chimneys/ snorkels often course obliquely across the neck. Wrapping, and hence seal, is particularly inefficient in these instances. 
The problem is worsened with multiple chimneys/snorkels. There is no consensus as to how to minimize gutter leaks. Although several authors have advocated for their particular technique, optimal sizing, anatomic criteria, stent graft dimensions, configuration, types of stents and the number of chimneys/snorkels remain variable between published works and individual operators.

Although there exists little standardization of techniques, there are several popularized techniques within retrospective cohorts. The combination of Endurant (Medtronic, Santa Rosa CA) with Advanta/iCast (Atrium Medical, Hudson $\mathrm{NH}$ ) balloon expandable stent grafts has been studied within the PROTAGORAS study (2). Donas et al. found that their group was able to achieve $100 \%$ technical success, with statistically significant decreases in aortic sac diameters noted at a median follow up of two years (2). Indeed, within the large multicenter PERICLES registry, this combination appears to provide superior survival and protection from late type 1a endoleaks when compared to other chimney/ snorkel configurations (3). The highest risk of type 1 endoleak appears to occur with self-expanding branch stent grafts, instead of balloon expandable stent grafts (3).

ChEVAR failure occurs more frequently when more branches are required. The risk of occlusion of the branch stent increases by almost two-fold with each additional chimney/snorkel (3). The risk of mortality also increases with each additional branch graft by more than three-fold (3). The individual endograft manufacturer does not appear to influence outcome significantly. Finally, the early type I leak rate is much higher than the late type I leak rate, which limits the application of ChEVAR in the setting of rupture.

\section{Comparisons of fenestrated/branched EVAR versus ChEVAR}

Comparisons of FEVAR versus ChEVAR are difficult. Bias by indication afflicts several series of ChEVAR, which limits ChEVAR to those ineligible for open or fenestrated repair. Moreover, device evolution, particularly with regards to fenestrated/branched endografts, has outpaced data abstraction. Most single-center cohort studies fail to stratify for baseline risks of complications, extent of visceral vessel involvement or size of the aneurysm. Finally, durability of ChEVAR and FEVAR are unclear given the relatively short follow up in the published literature.

Notwithstanding, there are several studies comparing FEVAR and ChEVAR. Most notably, O'Donnell et al. (4) used the Vascular Quality Initiative to evaluate stroke, death and major adverse cardiac events after fenestrated EVAR, PMEG and ChEVAR. ChEVAR patients had statistically significantly worse stroke and myocardial infarction rates. Other outcome differences were not statistically significant and were similar to outcomes described in prior series of complex EVARs. However, for all metrics, ChEVAR performed the worst, even when propensity matching the cohorts. Most concerningly is that ChEVAR had the worst perioperative mortality at $6.1 \%$ (4).

\section{Authors' opinions}

Neither ChEVAR nor FEVAR currently provide perfect solutions for all patients. Current data fail to conclusively determine a clearly superior endovascular approach. However, the existing comparative effectiveness research suggests some advantages to FEVAR compared to ChEVAR, particularly with stroke and survival rates. Moreover, device and technique evolution has been most significant for fenestrated/branched platforms in recent years, with more clinical outcomes and engineering data accumulating to show the efficacy of the most modern fenestrated platforms.

Although ChEVAR currently remains an acceptable solution, ChEVAR cannot surmount the limitation of gutter leaks without significant re-engineering and standardization of technique. This is particularly true when the branches course obliquely across the intended neck. Given the current trends in device and technique evolution, FEVAR will likely overtake ChEVAR in the near future as the dominant technology utilized to manage juxtarenal and type IV TAAA.

\section{Acknowledgments}

Funding: None.

\section{Footnote}

Conflicts of Interest: The authors declare no conflicts of interest.

Open Access Statement: This is an Open Access article distributed in accordance with the Creative Commons Attribution-NonCommercial-NoDerivs 4.0 International License (CC BY-NC-ND 4.0), which permits the noncommercial replication and distribution of the article with the strict proviso that no changes or edits are made and the 
original work is properly cited (including links to both the formal publication through the relevant DOI and the license). See: https://creativecommons.org/licenses/by-nc-nd/4.0/.

\section{References}

1. Lee JT, Greenberg JI, Dalman RL. Early experience with the snorkel technique for juxtarenal aneurysms. J Vasc Surg 2012;55:935-46; discussion 945-6.

2. Donas KP, Torsello GB, Piccoli G, et al. The PROTAGORAS study to evaluate the performance of the

Cite this article as: Pallister ZS, Milner R, Chung J. Snorkel and chimney endografting for juxtarenal and thoracoabdominal aortic aneurysms is an acceptable, but aging technology. Ann Cardiothorac Surg 2022;11(1):50-52. doi: 10.21037/acs-2021taes-14
Endurant stent graft for patients with pararenal pathologic processes treated by the chimney/snorkel endovascular technique. J Vasc Surg 2016;63:1-7.

3. Scali ST, Beck AW, Torsello G, et al. Identification of optimal device combinations for the chimney endovascular aneurysm repair technique within the PERICLES registry. J Vasc Surg 2018;68:24-35.

4. O'Donnell TFX, Patel VI, Deery SE, et al. The state of complex endovascular abdominal aortic aneurysm repairs in the Vascular Quality Initiative. J Vasc Surg 2019;70:369-80. 